\title{
Colorectal cancers survivors' adherence to lifestyle recommendations and cross-sectional associations with health-related quality of life
}

\author{
Jose J. L. Breedveld-Peters ${ }^{1 *}$, Janna L. Koole ${ }^{1}$, Eloise Müller-Schulte ${ }^{2} \dagger$, Bernadette W. A. van der Linden ${ }^{3} \ddagger$, \\ Cherelle Windhausen ${ }^{4} \S$, Martijn J. L. Bours ${ }^{1}$, Eline H. van Roekel ${ }^{1}$ and Matty P. Weijenberg ${ }^{1}$ \\ ${ }^{1}$ Department of Epidemiology, GROW - School for Oncology and Developmental Biology, Maastricht University, PO Box 616, \\ 6200 MD Maastricht, The Netherlands \\ ${ }^{2}$ Department of Epidemiology, GROW - School for Oncology and Developmental Biology, Maastricht University as part of \\ an internship from the Master of Global Health of the Faculty of Health, Medicine and Life Sciences, Maastricht University, PO \\ Box 616, 6200 MD Maastricht, The Netherlands \\ ${ }^{3}$ Department of Epidemiology, GROW - School for Oncology and Developmental Biology, Maastricht University as part of an \\ internship from the Health Sciences Research Master of the Faculty of Health, Medicine and Life Sciences, Maastricht \\ University, PO Box 616, 6200 MD Maastricht, The Netherlands \\ ${ }^{4}$ Department of Epidemiology, GROW - School for Oncology and Developmental Biology, Maastricht University as part of \\ an internship from the Master Epidemiology of the Faculty of Health, Medicine and Life Sciences, Maastricht University, PO \\ Box 616, 6200 MD Maastricht, The Netherlands
}

(Submitted 11 July 2017 - Final revision received 6 December 2017 - Accepted 2 January 2018 - First published online 16 April 2018 )

\section{Abstract}

The lifestyle recommendations of the World Cancer Research Fund (WCRF)/American Institute for Cancer Research (AICR) are primarily intended for cancer prevention. In the absence of specific recommendations for cancer survivors, we investigated adherence of colorectal cancer (CRC) survivors to the WCRF/AICR lifestyle recommendations and associations with health-related quality of life (HRQoL). The crosssectional part of the Energy for life after ColoRectal cancer (EnCoRe) study was conducted in 155 CRC survivors (stage I-III), 2-10 years post diagnosis. Dietary intake, physical activity and general body fatness were measured by 7 -d food diaries, by questionnaires and accelerometers and BMI, respectively. Adherence to each of the ten WCRF/AICR recommendations was scored as 0 (no/low adherence), 0.5 (moderate adherence) or 1 point (complete adherence), and summed into an overall adherence score (range: 0-10). HRQoL, disability and distress were assessed by validated questionnaires. Associations of the overall WCRF/AICR adherence score with HRQoL outcomes were analysed by confounder-adjusted linear regression. The mean adherence score was 5.1 (sD 1.4, range: 1.5-8.5). In confounder-adjusted models, a higher adherence score was significantly associated with the HRQoL dimension better physical functioning ( $\beta$ per 1 point difference in score: $2 \cdot 6$; $95 \%$ CI $0 \cdot 2,5 \cdot 1)$ and with less fatigue $(\beta:-3 \cdot 3 ; 95 \%$ CI $-6 \cdot 4,-0 \cdot 1)$. In conclusion, higher adherence of CRC survivors to WCRF/AICR lifestyle recommendations for cancer prevention was associated with better physical functioning and with less fatigue. This study adds to the limited knowledge on adherence to lifestyle behaviours in CRC survivors and relationships with quality of life. Prospective studies are needed to investigate longitudinal associations.

Key words: Lifestyle recommendations: Health-related quality of life: Colorectal cancer survivors

There is a steady increase in the number of colorectal cancer (CRC) survivors worldwide ${ }^{(1)}$. After being diagnosed with CRC, a healthy lifestyle may be highly relevant in terms of survival, recurrence and co-morbidities, as well as treatment-related health and functioning problems, and health-related quality of life (HRQOL) of CRC survivors ${ }^{(2)}$.
Results of a number of studies suggest that a healthy lifestyle after CRC diagnosis - for example maintaining a healthy body weight, being physically active on a regular basis and limiting the consumption of unhealthy foods such as red and processed meat and refined carbohydrates - may improve clinical outcomes including disease recurrence and survival ${ }^{(3)}$. In addition,

Abbreviations: CRC, colorectal cancer; EnCoRe, Energy for life after ColoRectal cancer; HRQoL, health-related quality of life; MVPA, moderate-to-vigorous physical activity; PA, physical activity; QoL, quality of life; WCRF/AICR, World Cancer Research Fund/American Institute for Cancer Research.

* Corresponding author: J. J. L. Breedveld-Peters, fax +31 4338 84128, email jose.breedveld@maastrichtuniversity.nl

$\dagger$ Present address: Center of Excellence for Microbiology and Hygiene, St. Franziskus Hospital, Münster, Germany.

$\ddagger$ Present address: Center for the Interdisciplinary Study of Gerontology and Vulnerability, University of Geneva, Geneva, Switzerland.

$\S$ Present address: Department of Dietetics, Treatment and Guidance Services, Envida, Maastricht, The Netherlands. 
a number of studies have shown significant associations of single lifestyle recommendations, such as physical activity $(\mathrm{PA})^{(4,5)}$ and obesity ${ }^{(5,6)}$, with HRQoL outcomes. However, to date, very little is known regarding associations of dietary factors with HRQoL in CRC survivors ${ }^{(7)}$. Moreover, only few studies have investigated associations of adherence to a comprehensive set of lifestyle recommendations with HRQOL in CRC survivors. Developed summary measures take multiple lifestyle factors into account and therefore give a good reflection of an overall lifestyle quality. These studies have usually been performed in mixed cancer survivor populations, including CRC and other cancers ${ }^{(8)}$.

The level of adherence to a combination of lifestyle recommendations in CRC survivors has been investigated in several studies. Lemasters et al. ${ }^{(9)}$ found that female CRC survivors shortly after CRC diagnosis were more likely to meet recommendations of fruit/vegetable intake and BMI, but less likely to meet alcohol and PA recommendations, in comparison with individuals without a history of cancer. Grimmett et $a l .{ }^{(7)}$ reported that the majority of long-term CRC survivors were overweight/obese, not physically active and not meeting fruit/vegetable recommendations. This is in line with findings of Schlesinger et $a l^{(10)}$, who reported that only $23 \%$ of long-term CRC survivors adhered to all four investigated lifestyle recommendations comprising BMI, healthy diet, PA and smoking. However, as these studies use different sets of lifestyle elements, comparison of results is difficult as the lifestyle factors used in the set differ between studies, as well as operationalisation of the lifestyle factors. Another way of looking at lifestyle is using existing lifestyle scores based on recommendations to investigate adherence to lifestyle behaviours in a more standardised way. In the absence of specific lifestyle recommendations for cancer survivors, the World Cancer Research Fund (WCRF)/American Institute for Cancer Research (AICR) currently advises cancer survivors to follow the lifestyle recommendations for the prevention of cancer ${ }^{(11,12)}$. These recommendations are based on extensive systematic reviews of the relevant literature on food, nutritional aspects, PA, body weight and cancer, and contain a range of lifestyle recommendations that can be operationalised into a lifestyle score that can be used to study overall lifestyle quality ${ }^{(13)}$. As the recommendations focus on body composition, PA and dietary factors, smoking is not part of this score. In CRC survivors, only one study of Winkels et al. ${ }^{(14)}$ used the WCRF/AICR lifestyle recommendations to study adherence to lifestyle recommendations. Thus, there is a need to increase knowledge on adherence of CRC survivors to an existing comprehensive set of lifestyle recommendations reflecting lifestyle quality and to study associations of lifestyle quality with outcomes of HRQOL in CRC survivors.

The aim of this cross-sectional study was to describe CRC survivors' adherence to the WCRF/AICR lifestyle recommendations for cancer prevention and to study how adherence is associated with relevant HRQOL outcomes, including global quality of life (QoL), physical functioning, fatigue, disability and distress. We hypothesised that a healthier lifestyle would be associated with better HRQoL.

\section{Methods}

\section{Study design and participants}

Data from the Energy for life after ColoRectal cancer (EnCoRe) study were used. The EnCoRe study is composed of two parts: a prospective cohort study in stage I-III CRC survivors followed up from diagnosis until 2 years post treatment, and a crosssectional study in stage I to III CRC survivors diagnosed and treated between 2002 and 2010 at Maastricht University Medical Center + , the Netherlands ${ }^{(15)}$. For the present analysis, data of the cross-sectional part of the EnCoRe study were used. Patients were preselected via the Netherlands Cancer Registry (NCR; managed by Comprehensive Cancer Centre the Netherlands). Participant recruitment and data collection were performed between May 2012 and December 2013. A total of 373 eligible CRC survivors were invited to participate, of whom 155 were included (response rate $42 \%$; online Supplementary Fig. S1). Reasons for exclusion of potential participants are shown in the online Supplementary Fig. S1. Ethical approval was obtained from the Medical Ethics Committee of Maastricht University and University Hospital Maastricht, the Netherlands, and written informed consent was obtained from all participants.

To systematically investigate lifestyle and HRQOL in CRC survivors, we have previously developed a conceptual framework $^{(15)}$ based on the International Classification of Functioning, Disability and Health (ICF). The ICF is a biopsychosocial classification of health and functioning developed by the $\mathrm{WHO}^{(16)}$. Relevant factors were identified from the literature and mapped within the ICF framework; this conceptual model was used to identify relevant variables to be included in the present analysis ${ }^{(15)}$.

\section{Data collection and data processing}

Study data were collected at patients' homes by trained research dietitians, according to standard operating procedures.

\section{World Cancer Research Fund/American Institute for Cancer Research lifestyle recommendations, score construction and operationalisation}

The WCRF/AICR lifestyle recommendations comprise recommendations on aspects of body composition, PA and diet. In this study, we included the WCRF/AICR recommendations regarding body fatness, PA, energy-dense foods, plant foods, meat consumption, alcoholic drinks and dietary supplements. We did not operationalise the salt restriction recommendation, as it is difficult to assess salt intake and participants were not requested to specifically report the addition of salt to meals. In addition, we did not operationalise the breast-feeding recommendation as it was not relevant for this population ${ }^{(11)}$. An overview of the applied WCRF/AICR lifestyle (sub)recommendations, as well as the operationalisation of the recommendations, and scoring thereof are shown in Table 1.

The first recommendation is to limit body fatness by being as lean as possible without becoming underweight. To operationalise the body fatness recommendation, body height $(\mathrm{m})$ and weight ( $\mathrm{kg})$ measurements were used to calculate BMI $\left(\mathrm{kg} / \mathrm{m}^{2}\right)$, and the predefined WHO categories were used, as shown in Table 1. 
Table 1. Operationalisation* of lifestyle recommendations for the prevention of cancer by the World Cancer Research Fund and American Institute for Cancer Research (WCRF/AICR)

(Numbers and percentages of participants with high, moderate and low adherence to recommendations)

\section{WCRF/AICR cancer prevention}

recommendations†

Study data

Opera
Normal
weight
Overweight
Obese
High
Moderate
Low
Low§
Moderate
High
Low
Moderate

ationalisation

Lifestyle score $n(n 145) \quad \%$

1. Be as lean as possible without becoming underweight; maintain body weight within the normal range

2a. Be physically active as part of your everyday life; be moderately physically active for $\geq 30$ min every day

$2 b$. Be physically active as part of everyday life; limit sedentary habits

3a. Limit consumption of energy-dense foods; consume energy-dense foods

BMI
Level of moderate-to-
vigorous physical activity
(MVPA)
Level of prolonged sedentary
behaviour (in bouts
of $\geq 30$ min)
Energy density of foods
consumed
sparingly

3b. Limit consumption of energy-dense foods; avoid sugary drinks

4a. Eat mostly foods of plant origin. Eat $\geq 400 \mathrm{~g}$ of a variety of non-starchy fruits and vegetables every day

4b. Eat mostly foods of plant origin; eat relatively unprocessed cereals (grains) and/or pulses (legumes)

5. Limit intake of red meat and avoid processed meat. Consume $<500 \mathrm{~g}$ red meat/week and very few, if any processed meats

Sugary drink consumption

Fruit and vegetable intake

Dietary fibre intake

\section{Dietary intake of red meat} and processed meat

$\begin{array}{cc} & \text { per d } \\ \text { High } & \geq 735 \mathrm{~kJ} / 100 \mathrm{~g} \mathrm{per} \mathrm{d} \\ \text { Low } & 0 \mathrm{~g} / \mathrm{d} \\ \text { Moderate } & >0 \text { and } \leq 250 \mathrm{~g} / \mathrm{d} \\ \text { High } & >250 \mathrm{~g} / \mathrm{d} \\ \text { High } & \geq 400 \mathrm{~g} / \mathrm{d} \\ \text { Moderate } & \geq 200-<400 \mathrm{~g} / \mathrm{d} \\ \text { Low } & <200 \mathrm{~g} / \mathrm{d} \\ \text { High } & \geq 25 \mathrm{~g} / \mathrm{d} \\ \text { Moderate } & \geq 12.5-<25 \mathrm{~g} / \mathrm{d} \\ \text { Low } & <12.5 \mathrm{~g} / \mathrm{d} \\ \text { Low } & <500 \mathrm{~g} \mathrm{red} \mathrm{meat/week} \\ & \text { and }<3 \mathrm{~g} \mathrm{processed} \\ & \text { meat } / \mathrm{d}\end{array}$

Moderate $<500 \mathrm{~g}$ red meat/week and $\geq 3-<50 \mathrm{~g}$ processed meat/d High $\quad \geq 500 \mathrm{~g} \mathrm{red}$ meat $/$ week or $\geq 50 \mathrm{~g}$ processed meat/d

6. Limit alcoholic drinks. If alcoholic drinks are consumed, limit consumption to $\leq 2$ drinks/d for men and $\leq 1 \mathrm{drink} / \mathrm{d}$ for women

7. Meet nutritional needs through diet alone; Dietary supplement use dietary supplements are not recommended for cancer prevention

Total score of 10 operationalised (sub) recommendations
Intake of alcohol

$$
0 \leq 20 \mathrm{~g}, \text { } \% \leq 10 \mathrm{~g} / \mathrm{d}
$$$$
\text { ot }>20-\leq 30 \mathrm{~g} / \mathrm{d} \text {, }
$$$$
q \geq 10-\leq 20 \mathrm{~g} / \mathrm{d}
$$

Moderate

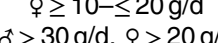

High

No dietary supplement use

Yes Dietary supplement use Minimum-maximum

1

138

$38 \quad 26 \cdot 2$

0.5

1

0.5

0

1

0.5

0

1

0.5

$66 \quad 45.5$

$41 \quad 28.3$

$\begin{array}{ll}71 & 49.0\end{array}$

$\begin{array}{rr}2 & 1.4 \\ 72 & 49.7\end{array}$

1.4
49.7

29.7

49.0

$21 \cdot 4$

21.4
$4 \cdot 1$

$62 \cdot 1$

$\begin{array}{lll}0 & 49 & 33.8\end{array}$

$\begin{array}{lll}1 & 48 & 33.1\end{array}$

$\begin{array}{lll}0.5 & 85 & 58.6\end{array}$

$\begin{array}{lll}0 & 12 & 8.3\end{array}$

$\begin{array}{lll}1 & 27 & 18.6\end{array}$

$\begin{array}{lll}0.5 & 78 & 53.8\end{array}$

$\begin{array}{lll}0.5 & 40 & 27.6\end{array}$

$\begin{array}{lll}0 & 43 & 29.7\end{array}$

$\begin{array}{lll}0.5 & 91 & 62.8\end{array}$

$\begin{array}{lll}0 & 11 & 7.6\end{array}$

$\begin{array}{lll}1 & 2 & 1.4\end{array}$

$0.5 \quad 50 \quad 34.5$

0

93

0.5

$64 \cdot 1$

0

17.9

0 $0-10$

PA, physical activity; ${ }^{\star}$, men; , women; CRC, colorectal cancer.

* Operationalisation used by Romaguera ${ }^{(17)}$ unless specified otherwise

† The WCRF/AICR 2007 recommendations have been used. Three of the (sub) recommendations are not operationalised in this score: we did not operationalise the fast food and the starchy food recommendation as there is currently no international consensus on the definitions for fast foods and for starchy foods, and these items overlap with the operationalised other items with respect to energy density ( $3 a$ and $3 b)$, and plant foods ( $4 a$ and $4 b$ ). Also the recommendation to limit salt consumption was not included in the score, as the amount of especially added salt during meal preparation is difficult to measure, and not available in the study, and since this is less relevant to CRC because there is no evidence for a role of salt in CRC aetiology.

$\ddagger$ There was one person with a $\mathrm{BMI}<18.5 \mathrm{~kg} / \mathrm{m}^{2}$. This person was included in this category.

$\S$ Cut-off values are based on what has been previously used in studies relating self-reported sedentary behaviour to risk of colon and rectal cancer literature by Lynch et al. ${ }^{\text {(23) }}$, Cong et al..$^{(24)}$ and Howard et al. ${ }^{(25)}$.

The second recommendation is to be physically active as part of one's daily life. PA was assessed by self-report, as well as using accelerometers. Self-reported PA was measured using the Short QUestionnaire to ASsess Health-enhancing physical activity, which includes frequency (d/week), duration (time/d) and intensity (light, moderate or vigorous) of commuting, household, work and leisure activities in the previous week. On the basis of assigned metabolic equivalent (MET) values (where 1 MET is defined as $1 \mathrm{kcal} / \mathrm{kg}$ per $\mathrm{h}$ ) and the intensity reported, activities were categorised as light or moderate-to-vigorous physical activity (MVPA). Data on weekly MVPA levels were used for classification into adherence score categories according to the PA guidelines for the
Dutch general population, which advises individuals to engage in at least $30 \mathrm{~min} / \mathrm{d}$ of MVPA on at least $5 \mathrm{~d} / \mathrm{week}^{(19)}$.

Accelerometers were used for obtaining objective data on sedentary behaviour. Thigh-mounted tri-axial MOX activity monitors $^{(20,21)}$ (MMOXX1; Maastricht Instruments B.V.) were worn by participants $24 \mathrm{~h} / \mathrm{d}$ during 7 consecutive days. Data were collected and processed as previously described ${ }^{(22)}$. Participants were required to report sleep or any non-wear periods, and these were excluded from the data. As there are, to our knowledge, currently no public recommendations available for sedentary behaviour, the cut-off values used for classification into adherence categories were based on what has been 
previously applied in studies relating self-reported sedentary behaviour to cancer risk ${ }^{(23-25)}$

The dietary recommendations are to avoid excessive energy intake by limiting energy-dense foods and sugary drinks; to eat mostly plant foods by having a higher intake of fruit, vegetables and dietary fibre; to limit animal food products by reducing red and processed meat intake; to limit consumption of alcoholic drinks; to limit salt intake; to not use dietary supplements; and for mothers to breast-feed their children. For the dietary recommendations data, we used quantitative data on $7-\mathrm{d}$ food intake assessed by dietary records. Methods and procedures applied for the assessment and coding of dietary records are explained in detail in the Supplementary Material S2. Participants were asked to fill out a structured dietary record to quantitatively assess food and drink consumption on 7 consecutive days. Dietary records were coded by trained dietitians, and 7-d mean daily intake of foods, macronutrients and micronutrients will be available for data analyses.

The WCRF/AICR dietary recommendation scores were calculated on the basis of the Dutch Food Composition table data, using corresponding existing or created dietary groups within Compl-eat (e.g. for red meat, processed meat, sugary drinks, fruits, vegetables). Energy density $(\mathrm{kJ} / 100 \mathrm{~g})$ was calculated from the energy provided by all solid and semi-solid foods. Sugary drinks (g/d) were calculated as the amount of sugar-containing drinks used. Fruit and vegetable consumption $(\mathrm{g} / \mathrm{d})$ were calculated from the reported use of all fresh, frozen, dried and canned vegetables and fruit without added sugar. Red meat consumption $(\mathrm{g} / \mathrm{d})$ was based on intake of any kind of fresh raw red meat that still needed to be prepared before consumption. Intake of processed meat $(\mathrm{g} / \mathrm{d})$ included intake of any meat that had been preserved and was ready for consumption. Calculation of total dietary fibre intake $(\mathrm{g} / \mathrm{d})$ was based on the nutrient value from the food calculation table for total fibre $(\mathrm{g} / \mathrm{d})$ according to the reported dietary intake. The same approach was used for alcohol intake $(\mathrm{g} / \mathrm{d})$.

Dietary supplement data were collected during home visits, through structured face-to-face interviews of the dietitians with participants. Information on supplement use, type and brand, ingredients, dosage and frequency of use was recorded and registered in detail. The original package of the supplement was used by the dietitians during the home visits to record type, brand and ingredients. For operationalisation of this recommendation, we used two score categories corresponding with supplement use or no dietary supplement use (see Table 1).

To finally calculate the WCRF/AICR adherence score, each recommendation was assigned a score of 1 point for complete adherence, 0.5 points for moderate adherence and 0 points for nonadherence. Predefined cut-off values of the WCRF/AICR recommendations adherence categories were used ${ }^{(26)}$. By summing the scores of the ten operationalised (sub) recommendations, an overall adherence score for the WCRF/AICR lifestyle recommendations was calculated (score range: 0-10 points). A higher score thus indicated a higher adherence to lifestyle recommendations.

\section{Additional analyses with the Dutch Healthy Diet Index}

As the Dutch CRC survivors in this study were mainly exposed to Dutch dietary recommendations, we performed extra analyses to investigate adherence to the national Dutch recommendations for a Healthy Diet (DHD) and associations with HRQoL outcomes. Adherence to these recommendations was scored using the previously published Dutch Healthy Diet Index score $(\mathrm{DHD}-\mathrm{i})^{(27)}$, which is a continuous score with ten components that represent the Dutch Recommendations for a healthy Diet (2006) comprising one PA component and nine dietary recommendations. The dietary recommendations are as follows: (1) eat 150-200 g of vegetables/d; (2) eat $200 \mathrm{~g}$ of fruit/ $\mathrm{d}$ and up to a maximum of $100 \mathrm{~g}$ of fruit juices that naturally contain folate and vitamin C; (3) eat 30-40 g/d of dietary fibre; (4) eat two portions of fish a week; (5) limit SFA consumption to $<10 \%$ of energy intake; (6) limit consumption of table salt to $6 \mathrm{~g} / \mathrm{d}$; (7) if alcohol is consumed at all, intake should be limited to two Dutch units ( $20 \mathrm{~g}$ ethanol) a day for males and one for females; (8) limit mono-trans-fatty acid consumption to $<1 \%$ of energy intake; (9) limit the consumption of foods and beverages that contain easily fermentable sugars and drinks. Methods and results of the DHD-i are presented in the online Supplementary Tables S3(a)-(b).

\section{Health-related quality of life}

HRQoL outcomes were measured by self-report using validated and reliable questionnaires. The European Organization for the Research and Treatment of Cancer Quality of Life Questionnaire-Core 30 (EORTC QLQ-C30, version 3.0 ${ }^{(28)}$ ) was used to assess cancer-specific HRQoL. For EORTC QLQ-C30 subscales, including global QoL, and physical, role and social functioning, 100-point scores were calculated, with higher scores indicating higher levels of QoL and functioning. The CRC-specific EORTC module CR29 was used to identify gastrointestinal problems (constipation, diarrhoea, abdominal pain, buttock pain, bloating, blood and mucus in stool, flatulence, faecal incontinence and sore skin). The twelve-item WHO Disability Assessment Schedule II (WHODAS II) was used to measure disability. A weighted disability score (100point scale) was computed, with a higher score indicating higher levels of disability. The Checklist Individual Strength (CIS $)^{(29,30)}$ was used to measure self-reported fatigue and the Hospital Anxiety Depression Scale (HADS) ${ }^{(31)}$ was used to measure emotional distress. Individual items from the CIS and HADS were summed to compute a total score for fatigue (range 20-140) and distress (range 0-42), respectively, with higher scores indicating higher levels of fatigue and distress.

\section{Other factors}

Socio-demographic characteristics (sex, age, education level, and smoking status) and presence of stoma were self-reported. The presence of co-morbidities was assessed using the SelfAdministered Comorbidity Questionnaire ${ }^{(32)}$. Data on clinical characteristics (cancer stage, age at diagnosis, treatment) were collected through the Netherlands Cancer Registry.

\section{Statistical analyses}

In all, ten survivors were excluded from the present analyses owing to missing HRQoL data ( $n$ 2), insufficient number 
of dietary measurement days ( $n$ 2), incorrectly calibrated accelerometers ( $n$ 2) or missing accelerometer data $(n 4)$. This resulted in the inclusion of a final number of 145 stage I-III CRC survivors (ninety-one male and fifty-four female) in the present analyses.

Descriptive analyses of socio-demographic and clinical characteristics of participants were performed. The adherence of participants to the individual WCRF/AICR recommendations was calculated for the overall population and stratified by sex. Group means and standard deviations were compared for continuous variables and absolute and relative frequencies for categorical variables. Significant differences in WCRF/AICR lifestyle scores between different groups (e.g. sex, BMI categories, tumour stage and so on) were assessed using independent samples $t$ tests or ANOVA depending on whether there were two or more than two categories for the groups, respectively.

Confounder-adjusted linear regression models were used to assess associations between the continuous WCRF/AICR adherence score and HRQoL outcomes. Main HRQOL outcomes included global QoL, physical functioning, role functioning and social functioning (EORTC QLQ-C30), disability (WHODAS II), fatigue (CIS) and distress (HADS). Unstandardised regression coefficients $(\beta)$ and $95 \%$ CI were obtained from linear regression models for each of the HRQoL outcomes, representing the difference in mean HRQOL scores per one-point increment in the WCRF adherence score. Because some HRQOL outcomes were not normally distributed, sensitivity analyses by means of logistic regression models with dichotomised outcomes were previously conducted and showed results similar to linear regression analyses ${ }^{(22,33)}$.

A priori-defined confounders included in the analyses were age (years), sex, number of co-morbidities ( $0,1,2$ or more), smoking (current, non-current), education level (low, medium, high), tumour stage (I, II, III), chemotherapy treatment (yes, no), time since diagnosis (years), total energy intake (kJ/week (kcal/week)), stoma (yes/no) and presence of any of the gastrointestinal problems such as constipation, diarrhoea, abdominal pain, buttock pain, bloating, blood and mucus in stool, flatulence, faecal incontinence and sore skin (yes/no). Regarding the sample size for linear regression models, we applied the rule of thumb to use a minimum of ten participants per independent variable included in the model ${ }^{(34)}$.

In addition, we performed stratified analyses to explore whether associations differed by sex, BMI (normal weight $v$. overweight/obesity) and co-morbidities $(0-1 \quad v .2$ or more co-morbid conditions). Next, as previous research in this study population yielded significant associations of $\mathrm{PA} /$ sedentary behaviour and several HRQoL outcomes ${ }^{(22,33,35)}$, additional regression analyses were performed excluding the PA recommendations from the WCRF/AICR score, to determine whether observed associations were driven by the PA or sedentary behaviour components of the score. Furthermore, regression analyses were also performed with tertiles of the WCRF adherence score to compare survivors with high adherence with survivors with low adherence with regard to HRQOL outcomes.

Statistical analyses were carried out using IBM SPSS Statistics (version 22; IBM Corporation) with statistical significance being defined as $P<0.05$ (two-tailed testing).

\section{Results}

\section{Participant characteristics}

Participants were on average 70 years of age (SD 8.7 ) and the mean survival time since CRC diagnosis was 5.7 years (SD 1.9, range 2-9). As previously reported, study participants were on average younger, more often diagnosed with rectal cancer and had more frequently received chemotherapy and radiotherapy treatment compared with non-participants ${ }^{(22,33,35)}$. Participants' characteristics are shown in Table 2.

\section{Adherence to World Cancer Research Fund/American Institute for Cancer Research recommendations}

The mean WCRF/AICR adherence score was 5.1 (SD 1.4), ranging from 1.5 to 8.5 points. The degree of adherence to the individual recommendations is depicted in Fig. 1. In total, $28 \%$ of the population complied with the recommendation for a healthy BMI. Results for PA showed that about half of the population reported to adhere to the MVPA recommendation, and that about $30 \%$ adhered to the recommendation to limit sedentary behaviour. There was a large variation in adherence to the different dietary recommendations. Only $4 \%$ adhered to the recommendation to limit intake of energy-dense foods, $33 \%$ of survivors reported to adhere to the advice to limit sugary drink intake and moderate adherence was mainly reported for several other dietary recommendations. Fruit and vegetable intake recommendations were met by $19 \%$ of the population, whereas the majority ( $57 \%$ ) had a moderate adherence to fibre intake recommendations. Only $1 \%$ of participants reported to adhere to the recommendation to limit red meat intake and avoid processed meat, whereas on the other hand $64 \%$ reported full adherence to alcohol intake recommendations, and a considerable percentage (57\%) of survivors complied with the recommendation not to use dietary supplements. Differences in mean WCRF/AICR scores by sex are shown in the online Supplementary Table S4.

\section{Associations of World Cancer Research Fund/American Institute for Cancer Research adherence score with health-related quality of life}

In confounder-adjusted multivariable linear regression models, higher WCRF/AICR adherence score was significantly associated with better physical functioning ( $\beta: 2 \cdot 6 ; 95 \%$ CI $0 \cdot 2,5 \cdot 1)$ and with less fatigue $(\beta$ : $-3 \cdot 3 ; 95 \%$ CI $-6 \cdot 4,-0 \cdot 1)$ for the total group (Table 3). Associations of the overall score with other HRQOL outcomes were non-significant. Stratified analyses by sex showed that the overall score was significantly associated with better global QoL ( $\beta$ : 4.8; $95 \%$ CI $0.5,9 \cdot 2)$, better physical functioning $(\beta: 7 \cdot 4 ; 95 \%$ CI $2 \cdot 9,12 \cdot 0)$, less fatigue $(\beta$ : $-6 \cdot 0 ; 95 \% \mathrm{CI}-11 \cdot 8,-0 \cdot 1)$ and less disability $(\beta:-4 \cdot 4 ; 95 \%$ CI $-8 \cdot 2,-0 \cdot 6)$ in women only. In overweight/obese survivors, a higher overall score was significantly associated with better global QoL ( $\beta: 3 \cdot 1 ; 95 \%$ CI $0 \cdot 3,5 \cdot 9)$, better physical functioning $(\beta: 5 \cdot 0 ; 95 \%$ CI $2 \cdot 0,8 \cdot 1)$, less fatigue $(\beta:-5 \cdot 5 ; 95 \% \mathrm{CI}-9 \cdot 3,-1 \cdot 6)$, less disability $(\beta$ : $-3 \cdot 1 ; 95 \% \mathrm{CI}-5 \cdot 5,-0 \cdot 8)$ and less distress ( $\beta$ : $-1 \cdot 2 ; 95 \%$ CI $-2 \cdot 4,-0 \cdot 1)$. These associations were 
Table 2. Adherence scores for the World Cancer Research Fund and American Institute for Cancer Research (WCRF/AICR) lifestyle recommendations, socio-demographic and clinical characteristics

(Mean values and standard deviations; numbers and percentages)

\begin{tabular}{|c|c|c|c|c|c|c|}
\hline & \multicolumn{2}{|c|}{ Total group ( $n$ 145) } & \multicolumn{2}{|c|}{ Men $(n$ 91, $63 \%)$} & \multicolumn{2}{|c|}{ Women ( $n$ 54, $37 \%)$} \\
\hline & Mean & SD & Mean & SD & Mean & SD \\
\hline \multicolumn{7}{|l|}{ Range } \\
\hline WCRF/AICR adherence score $(0-10)$ & \multicolumn{2}{|c|}{$1.5-8.5$} & \multicolumn{2}{|c|}{$2.5-8.5$} & \multicolumn{2}{|c|}{$1.5-8.0$} \\
\hline WCRF/AICR adherence score & $5 \cdot 1$ & 1.4 & $5 \cdot 1$ & 1.3 & 5.0 & 1.5 \\
\hline Age (years) & $70 \cdot 0$ & 8.7 & 69.9 & 8.0 & $70 \cdot 0$ & 9.8 \\
\hline Time since diagnosis (years) & $5 \cdot 7$ & 1.9 & 5.8 & 1.9 & 5.6 & 1.8 \\
\hline $\mathrm{BMI}\left(\mathrm{kg} / \mathrm{m}^{2}\right)$ & $27 \cdot 6$ & 4.3 & $27 \cdot 4$ & 3.4 & $28 \cdot 1$ & 5.6 \\
\hline \multicolumn{7}{|l|}{ Physical activity level (h/d) } \\
\hline LPA $(\mathrm{h} / \mathrm{d})$ & $2 \cdot 1$ & 2.5 & 1.7 & $2 \cdot 6$ & $2 \cdot 9$ & $2 \cdot 1$ \\
\hline MVPA (h/d) & 1.8 & $2 \cdot 1$ & $2 \cdot 3$ & 2.5 & $1 \cdot 1$ & 0.9 \\
\hline Total PA (h/d) & 4.0 & $3 \cdot 2$ & 4.0 & $3 \cdot 6$ & 4.0 & $2 \cdot 3$ \\
\hline \multirow[t]{2}{*}{ Total energy intake $(\mathrm{kJ} / \mathrm{d})$} & 8234.9 & $2019 \cdot 8$ & $9169 \cdot 9$ & $1750 \cdot 6$ & 6659.9 & $1361 \cdot 6$ \\
\hline & $n$ & $\%$ & $n$ & $\%$ & $n$ & $\%$ \\
\hline \multicolumn{7}{|l|}{ BMI $\left(\mathrm{kg} / \mathrm{m}^{2}\right)$} \\
\hline$\leq 24.99$ & 39 & $26 \cdot 9$ & 21 & $23 \cdot 1$ & 18 & $33 \cdot 3$ \\
\hline $25 \cdot 00-29.99$ & 66 & $45 \cdot 5$ & 52 & $57 \cdot 1$ & 14 & $25 \cdot 9$ \\
\hline$\geq 30$ & 40 & $27 \cdot 6$ & 18 & $19 \cdot 8$ & 22 & 40.7 \\
\hline \multicolumn{7}{|l|}{ Number of co-morbidities } \\
\hline 0 & 35 & $24 \cdot 1$ & 24 & $26 \cdot 4$ & 11 & $20 \cdot 4$ \\
\hline 1 & 36 & $24 \cdot 8$ & 24 & $26 \cdot 4$ & 12 & $22 \cdot 2$ \\
\hline$\geq 2$ & 74 & $51 \cdot 0$ & 42 & $47 \cdot 3$ & 31 & 57.4 \\
\hline \multicolumn{7}{|l|}{ Smoking } \\
\hline Current & 16 & 11.0 & 12 & $13 \cdot 2$ & 4 & 7.4 \\
\hline Non-current & 129 & 89.0 & 79 & $86 \cdot 8$ & 50 & $92 \cdot 6$ \\
\hline \multicolumn{7}{|l|}{ Education level } \\
\hline Low & 37 & $25 \cdot 5$ & 19 & $20 \cdot 9$ & 18 & $33 \cdot 3$ \\
\hline Medium & 48 & $33 \cdot 1$ & 28 & $30 \cdot 8$ & 20 & $37 \cdot 0$ \\
\hline High & 60 & 41.4 & 44 & 48.4 & 16 & 29.6 \\
\hline \multicolumn{7}{|l|}{ Tumour location } \\
\hline Colon & 78 & 53.8 & 40 & 44.0 & 38 & $70 \cdot 4$ \\
\hline Rectosigmoid/rectum & 67 & $46 \cdot 2$ & 51 & $56 \cdot 0$ & 16 & 29.6 \\
\hline \multicolumn{7}{|l|}{ Tumour stage* } \\
\hline Stage 1 & 40 & $29 \cdot 2$ & 24 & 28.9 & 16 & 29.6 \\
\hline Stage 2 & 50 & 36.5 & 30 & $36 \cdot 1$ & 20 & 37.0 \\
\hline Stage 3 & 47 & $34 \cdot 3$ & 29 & 34.9 & 18 & $33 \cdot 3$ \\
\hline \multicolumn{7}{|l|}{ Cancer treatment } \\
\hline No chemotherapy & 70 & $48 \cdot 3$ & 43 & $47 \cdot 3$ & 27 & $50 \cdot 0$ \\
\hline Chemotherapy (yes) & 75 & 51.7 & 48 & $52 \cdot 7$ & 27 & $50 \cdot 0$ \\
\hline \multicolumn{7}{|l|}{ Stoma } \\
\hline No & 121 & 83.4 & 76 & 83.5 & 45 & $83 \cdot 3$ \\
\hline Yes & 24 & $16 \cdot 6$ & 15 & $16 \cdot 5$ & 9 & $16 \cdot 7$ \\
\hline \multicolumn{7}{|l|}{ Gastrointestinal problems $\dagger$} \\
\hline Yes & 54 & 37.8 & 32 & $35 \cdot 2$ & 22 & $42 \cdot 3$ \\
\hline No & 89 & $62 \cdot 2$ & 59 & 64.8 & 30 & 57.7 \\
\hline
\end{tabular}

LPA, light physical activity; MVPA, moderate-to-vigorous physical activity; PA, physical activity; CRC, colorectal cancer.

* Calculations for tumour stage are based on 137 patients.

† Gastrointestinal problems included constipation, diarrhoea, abdominal pain, buttock pain, bloating, blood and mucus in stool, flatulence, faecal incontinence and sore skin and were measured by means of the CRC-specific European Organization for the Research and Treatment of Cancer Quality of Life Questionnaire-Core module CR29. If any of these problems were reported, gastrointestinal problems were scored as 'yes'.

non-significant in normal-weight survivors. No significant associations were found in analyses stratified by number of co-morbidities.

Removal of the PA and sedentary behaviour recommendations from the WCRF/AICR score attenuated the associations with physical functioning and with fatigue for the overall population, as well as most results in women (online Supplementary Table S4). However, in women, associations remained significant with better global QoL $(\beta: 8 \cdot 3 ; 95 \%$ CI $1.9,14.8)$, and in overweight/ obese survivors with better global Qol $(\beta$ : 4.3; $95 \%$ CI 0.8, 7.7), better physical functioning $(\beta: 4 \cdot 4 ; 95 \% \mathrm{CI} 0 \cdot 7,8 \cdot 2)$, less fatigue $(\beta:-5 \cdot 8 ; 95 \% \mathrm{CI}-10 \cdot 6,-1 \cdot 1)$ and less disability $(\beta:-3 \cdot 4 ; 95 \% \mathrm{CI}$ $-6 \cdot 3,-0 \cdot 7)$ (online Supplementary Table S5).

The online Supplementary Table S6 shows the results of analysis of score tertiles. For the overall group, the highest tertile of overall adherence score was associated with less fatigue $(\beta$ : $-11 \cdot 2 ; 95 \% \mathrm{CI}-21 \cdot 6,-0 \cdot 8)$; however, when comparing these results with the continuous analyses, no significant difference was found for physical functioning in this analysis. In women, a significant association was found for the highest tertile of overall score with better physical functioning ( $\beta$ : $18.5 ; 95 \%$ CI $2 \cdot 1,35 \cdot 0$ ), but not for the other HRQoL 


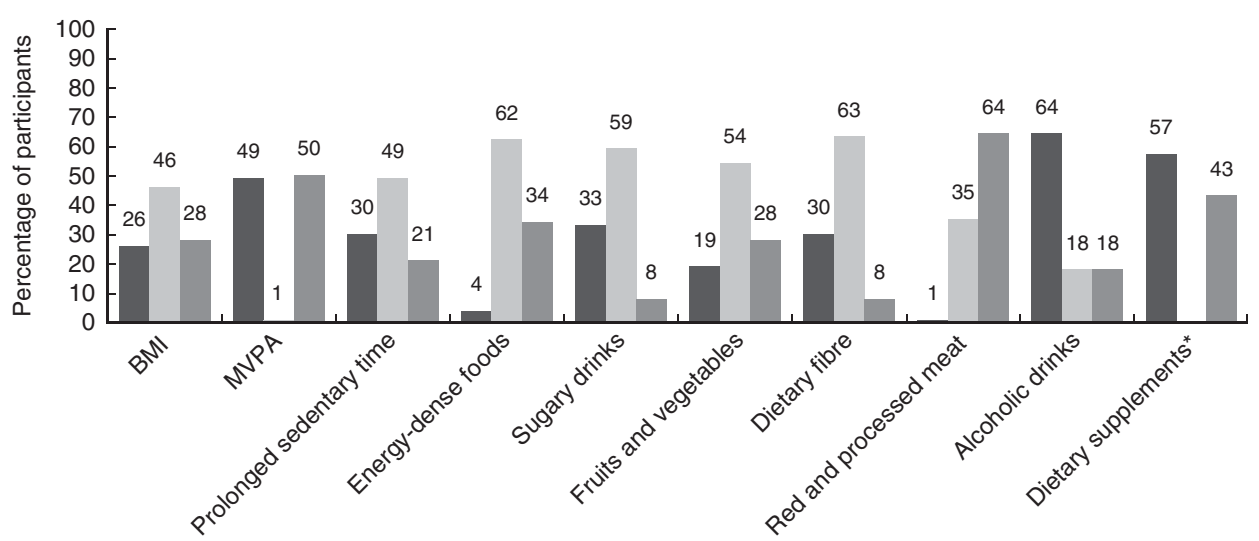

Fig. 1. Adherence to the individual World Cancer Research Fund/American Institute for Cancer Research (WCRF/AICR) lifestyle recommendations. $\square$, High adherence; $\square$, moderate adherence; $\square$, low adherence; MVPA, moderate-to-vigorous physical activity. * The recommendation for dietary supplements is operationalised using two score categories: the high-adherence category corresponds with no dietary supplement use, and the low-adherence category corresponds with participants with dietary supplement use.

outcomes for which we found associations in the continuous analyses. Results for overweight/obese survivors showed significant associations of the higher tertile of overall adherence score with better physical functioning ( $\beta$ : $10.5 ; 95 \%$ CI 0.9 , $-20 \cdot 0)$ and with less fatigue $(\beta:-13 \cdot 4 ; 95 \% \mathrm{CI}-25 \cdot 5,-1 \cdot 3)$, and not for the other outcomes for which significant associations were found in the continuous analyses.

\section{Results of the additional analyses with the Dutch Healthy} Diet 2006 index

Results of regression analyses of the DHD-i are shown in the online Supplementary Table S3(b), and were similar to those of the AICR/WCRF adherence score. We found significant associations of a higher DHD-i adherence score with better global QoL and physical functioning in the overall group; with better global QoL, better physical functioning and less disability in women only; and with better physical functioning in overweight/obese CRC survivors only (online Supplementary Table S3(b)).

\section{Discussion}

This cross-sectional study is one of the first studies investigating adherence of CRC survivors to the cancer prevention WCRF/AICR lifestyle recommendations and associations with HRQOL. The present study population of 155 CRC survivors most frequently had a moderate adherence to the ten studied lifestyle recommendations of WCRF/AICR. Higher adherence score was significantly associated with better physical functioning and less fatigue. Within subgroups of female survivors and overweight/ obese survivors only, higher adherence score was additionally associated with better global QoL and less disability. However, when the recommendations for PA and prolonged sedentary behaviour were excluded from the lifestyle score, most of the associations were attenuated. This suggests that PA and sedentary behaviour are important components of the lifestyle score with regard to associations with HRQOL. However, some associations remained significant and were less attenuated in women and obese/overweight survivors, suggesting that other lifestyle factors may also play an important role for HRQoL in these subgroups.

Several other studies in CRC survivors also reported moderate adherence to mainly investigator-constructed sets of lifestyle recommendations. This was observed regardless of the differences in the number and types of recommendations and the operationalisation of lifestyle factors included across stu$\operatorname{dies}^{(7,10,14)}$. In all, 30 and $38 \%$ of long-term CRC survivors from a cross-sectional study were reported to adhere to two and three out of four favourable lifestyle factors (non-obese, healthy diet, recreationally active and non-smoking), respectively ${ }^{(10)}$. Another study reported that the majority of CRC survivors 5 years after diagnosis were overweight/obese, not physically active and not adhering to fruit/vegetable recommendations ${ }^{(7)}$. Adherence to the WCRF/AICR recommendations in a Dutch study in CRC survivors ${ }^{(14)}$ showed similar results compared with the present study. We were able to operationalise an additional activity recommendation regarding prolonged sedentary behaviour based on objective accelerometer data, and additional dietary recommendations (i.e. consumption of energy-dense foods, sugary drink use, and foods of plant origin). Furthermore, we applied 7-d food diaries to measure dietary intake, whereas other studies used food frequency questionnaires. Food diaries enable a more extensive registration of dietary intake. Inclusion of additional recommendations in the score, as well as the use of more rigorous measurement instruments in our study, have probably resulted in a more comprehensive lifestyle score and better validity and reliability of data used for calculating the score.

Interestingly, we observed considerable variation in adherence levels across the individual WCRF/AICR recommendations, indicating that different individual score elements, and thus different individual lifestyle factors, contribute to the overall score of individuals. We found that the majority of participants had a moderate adherence for several individual lifestyle items of the score, including BMI, sedentary behaviour, sugary drink consumption and fruit and vegetable intake. However, adherence was very low for some other recommendations, such as the recommendation to limit red and processed meat consumption and consumption of energy-dense foods, 


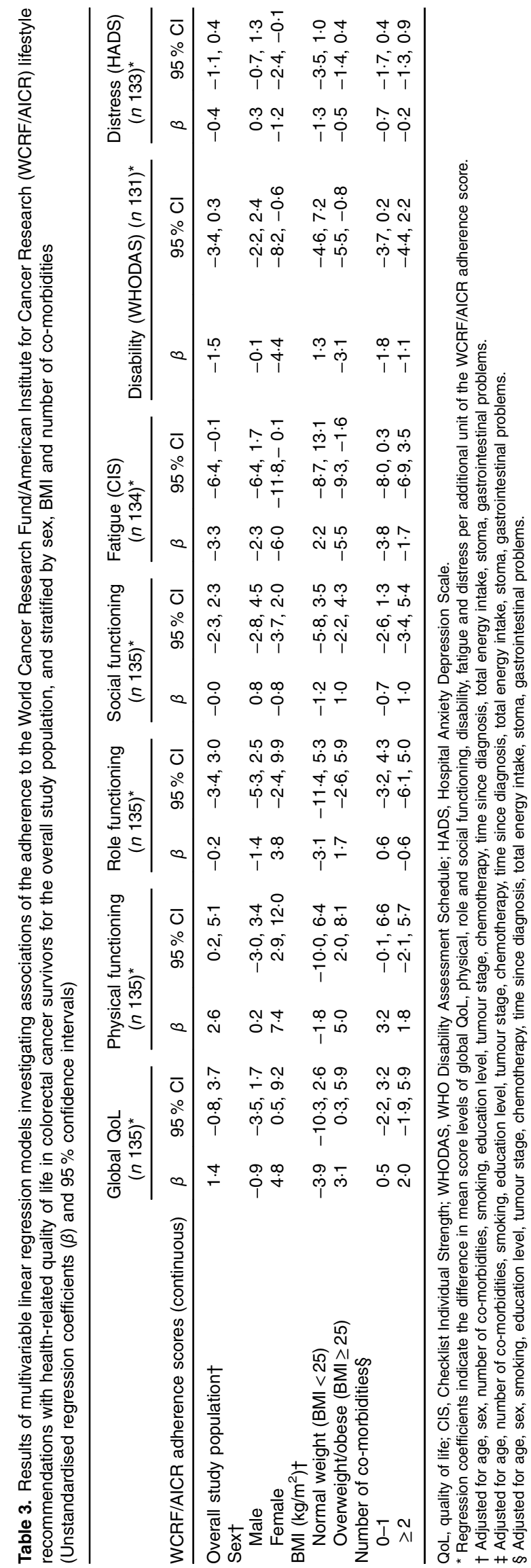

whereas on the other hand it was high for the recommendation to limit alcohol intake. Comparing our results with those of the PROFILES study ${ }^{(14)}$, they were similar for body fatness (BMI; despite differences in objective measurement $v$. self-reported assessment of body weight and height) and for the MVPA recommendation, but differed substantially for other recommendations as we found much lower levels of complete adherence to the recommendation to limit sugary drink intake (EnCoRe 33\%, v. Profiles 58\%), much lower levels of adherence to limit red and processed meat intake (EnCoRe 1\%, $v$. Profiles $8 \%$ ) and a lower level of adherence to the recommendation not to use dietary supplements (EnCoRe 57\%, $v$. Profiles $75 \%$ ). Differences may partly be explained by the use of other methods to measure dietary intake and by differences in populations included, as the general population in the southern part of the Netherlands is known to have a more unhealthy lifestyle compared with other parts of the Netherlands ${ }^{(36)}$. Altogether, our results and those of other studies show that there is ample room for improvement of lifestyle of CRC survivors.

To our knowledge, no other studies have investigated associations of lifestyle quality as measured with the WCRF/AICR recommendations with HRQOL in CRC survivors. Some studies investigated cross-sectional adherence to other sets of lifestyle recommendations and associations with HRQOL in CRC survivors. These studies showed that a more favourable health behaviour score was associated with better global QoL, physical functioning and less fatigue in CRC survivors diagnosed within the past 5 years $^{(7)}$, and that adherence to a higher number of recommended lifestyle factors was associated with better global HRQOL, and better physical, role and social functioning in longterm CRC survivors ${ }^{(10)}$. One study reported results of adherence to the WCRF/AICR lifestyle recommendations and associations with HRQOL in a specific group of elderly female CRC survivors $^{(37)}$. Our study also showed that higher adherence to an extensive lifestyle score incorporating several dietary items next to the components of body composition and PA is associated with better physical functioning and with less fatigue in the overall group of CRC survivors, especially in female and in overweight/obese survivors. This suggests that there is potential for lifestyle interventions for CRC survivors. Such lifestyle interventions would ideally be multifactorially directed at improvement of PA, diet quality and body weight (in overweight or obese survivors), to enhance different outcomes of QoL. Furthermore, to enable translation of results into practical recommendations, it is necessary to consider factors associated with (non)adherence to (WCRF/AICR) recommendations, which could guide the design, content and targeting of the interventions to specific groups. Although literature on factors influencing adherence to WCRF/AICR recommendations is limited, relevant factors could comprise, but are not limited to, physical factors, psychological factors and social factors ${ }^{(17,18)}$.

Strengths of our study included the use of a comprehensive existing set of lifestyle recommendations, as well as the use of more rigorous and objective measurement instruments (the dietary records, the accelerometer and objective anthropometric measurement). However, despite careful choice of assessment methods and attention to detail in analysis, some of 
the assessments are self-reported and hence may be subject to bias. In addition, it could be that, owing to selection bias, individuals with a low adherence to the lifestyle recommendations and low QoL and functioning and more fatigue were less likely to participate in the study. Therefore, the observed associations in our study may have been underestimated owing to selection bias. Furthermore, our study results have to be interpreted with caution, because of the relatively small sample size and because of the cross-sectional design of this study, which did not allow us to draw conclusions regarding the direction or causality of the observed associations. Participants' lifestyle behaviours and HRQOL were assessed at a single point in time, and thus we could not determine whether adherence to recommendations reflected long-term behavioural patterns. Results therefore have to be confirmed in longitudinal studies, such as the prospective part of our currently ongoing EnCoRe study.

In conclusion, this study adds to the limited knowledge base of adherence to lifestyle behaviours of CRC survivors and of relationships with HRQOL. Our results indicate that the majority of CRC survivors have a moderate adherence to the WCRF/ AICR lifestyle recommendations for cancer prevention and that adherence to individual lifestyle factors differ across lifestyle factors. Furthermore, our findings suggest that higher adherence to the WCRF/AICR lifestyle recommendations is associated with better physical functioning and less fatigue, especially in women and in overweight/obese survivors. Longitudinal studies are warranted to confirm our findings and to assess prospective relationships of a combination of lifestyle behaviours with HRQOL in CRC survivors.

\section{Acknowledgements}

The authors thank the coauthors Eloise Müller-Schulte, Bernadette W. A. van der Linden and Cherelle Windhausen for their valuable contribution to this study during their internships for the Master of Global Health, the Health Sciences Research Master and the Master Epidemiology, respectively, of the Maastricht University, The Netherlands. Furthermore, the authors would like to thank the EnCoRe study participants, the EnCoRe study research team (P. van der A-Wisselink, N. Wijckmans, D.O. Klein, Y.L.L. Vanlingen, C.M.J. Gielen, and A.G.E. Smeets), the registration teams of the Comprehensive Cancer Centre Netherlands and Comprehensive Cancer Centre South for the collection of data for the Netherlands Cancer Registry and the scientific staff of the Comprehensive Cancer Centre.

This study was supported by a grant from the Stichting Alpe d'HuZes within the research programme 'Leven met kanker' of the Dutch Cancer Society (grant no. UM-2010-4867). E. H. v. R. is financially supported by this grant. In addition, J. J. L. B.-P. is supported by a grant from Kankeronderzoekfonds Limburg (part of Health Foundation Limburg) (grant no. 00005739); M. J. L. B. is supported by a grant from the Stichting Alpe d'HuZes within the research programme 'Leven met kanker' of the Dutch Cancer Society (grant no. UM-2012-5653).

The authors' contributions were as follows: M. P. W. and M. J. L. B. formulated the research questions and designed the study; J. J. L. B.-P., J. L. K, E. H. v. R., M. J. L. B. and M. P. W. carried out the study; J. J. L. B.-P., J. L. K., E. M.-S., B. W. A. v. d. L., C. W., M. J. L. B., E. H. v. R. and M. P. W. were involved in analysing the data; and J. J. L. B.-P., J. L. K., E. M.-S., B. W. A. v. d. L., C. W., M. J. L. B., E. H. v. R. and M. P. W. wrote the article.

The first author J. J. L. B.-P. is presently employed at World Cancer Research Fund, the Netherlands (WCRF NL). The authors declare that there are no conflicts of interest.

\section{Supplementary material}

For supplementary material/s referred to in this article, please visit https://doi.org/10.1017/S0007114518000661

\section{References}

1. Hanly P, Walsh PM, O Céilleachair A, et al. (2013) Work-related productivity losses in an era of ageing populations: the case of colorectal cancer. J Occup Environ Med $\mathbf{5 5}$, $128-134$

2. Anderson AS, Steele R \& Coyle J (2013) Lifestyle issues for colorectal cancer survivors-perceived needs, beliefs and opportunities. Support Care Cancer 21, 35-42.

3. Van Blarigan EL \& Meyerhardt JA (2015) Role of physical activity and diet after colorectal cancer diagnosis. J Clin Oncol 33, 1825-1834.

4. Lynch BM, van Roekel EH \& Vallance JK (2016) Physical activity and quality of life after colorectal cancer: overview of evidence and future directions. Expert Rev Qual Life Cancer Care 1, 9-23.

5. Blanchard CM, Stein K \& Courneya KS (2010) Body mass index, physical activity, and health-related quality of life in cancer survivors. Med Sci Sports Exerc 42, 665-671.

6. van Roekel EH, Bours MJ, Te Molder ME, et al. (2017) Associations of adipose and muscle tissue parameters at colorectal cancer diagnosis with long-term health-related quality of life. Qual Life Res, 26, 1745-1759.

7. Grimmett C, Bridgewater J, Steptoe A, et al. (2011) Lifestyle and quality of life in colorectal cancer survivors. Qual Life Res 20, $1237-1245$.

8. Blanchard CM, Courneya KS, Stein K, et al. (2008) Cancer survivors' adherence to lifestyle behavior recommendations and associations with health-related quality of life: results from the American Cancer Society's SCS-II. J Clin Oncol 26, 2198-2204.

9. LeMasters TJ, Madhavan SS, Sambamoorthi U, et al. (2014) Health behaviors among breast, prostate, and colorectal cancer survivors: a US population-based case-control study, with comparisons by cancer type and gender. J Cancer Surviv $\mathbf{8}$, 336-348.

10. Schlesinger S, Walter J, Hampe J, et al. (2014) Lifestyle factors and health-related quality of life in colorectal cancer survivors. Cancer Causes Control 25, 99-110.

11. World Cancer Research Fund/American Institute for Cancer Research (2011) Continuous Update Project Report. Food, Nutrition, Physical Activity and the Prevention of Colorectal Cancer. WCRF/AICR. https://www.wcrf.org/sites/default/ files/Colorectal-Cancer-2011-Report.pdf

12. World Cancer Research Fund/American Institute for Cancer Research (2007)) Food, Nutrition, Physical Activity, and the Prevention of Cancer: a Global Perspective. Washington, DC: AICR.

13. Vergnaud AC, Romaguera D, Peeters PH, et al. (2013) Adherence to the World Cancer Research Fund/American 
Institute for Cancer Research guidelines and risk of death in Europe: results from the European Prospective Investigation into Nutrition and Cancer cohort study. Am J Clin Nutr 97, $1107-1120$

14. Winkels RM, van Lee L, Beijer S, et al. (2016) Adherence to the World Cancer Research Fund/American Institute for Cancer Research lifestyle recommendations in colorectal cancer survivors: results of the PROFILES registry. Cancer Med 5, $2587-2595$.

15. van Roekel EH, Bours MJ, de Brouwer CP, et al. (2014) The applicability of the international classification of functioning, disability, and health to study lifestyle and quality of life of colorectal cancer survivors. Cancer Epidemiol Biomarkers Prev 23, 1394-1405.

16. Chandler JC, Aizen J, Fitzgibbon QP, et al. (2016) Applying the power of transcriptomics: understanding male sexual development in decapod crustacea. Integr Comp Biol 56, 1144-1156.

17. Tsay SL, Ko WS \& Lin KP (2017) The lifestyle change experiences of cancer survivors. J Nurs Res 25, 328-335.

18. Humpel N, Magee C \& Jones SC (2007) The impact of a cancer diagnosis on the health behaviors of cancer survivors and their family and friends. Support Care Cancer 15, 621-630.

19. Comprehensive Cancer Center the Netherlands (2017) Guideline cancer rehabilitation. www.oncoline.nl/oncologischerevalidatie (accessed May 2017).

20. Berendsen BA, Hendriks MR, Meijer K, et al. (2014) Which activity monitor to use? Validity, reproducibility and user friendliness of three activity monitors. BMC Public Health 14, 749.

21. Annegarn J, Spruit MA, Uszko-Lencer NH, et al. (2011) Objective physical activity assessment in patients with chronic organ failure: a validation study of a new single-unit activity monitor. Arch Phys Med Rehabil 92, 1852-7 e1.

22. van Roekel EH, Winkler EA, Bours MJ, et al. (2016) Associations of sedentary time and patterns of sedentary time accumulation with health-related quality of life in colorectal cancer survivors. Prev Med Rep 4, 262-269.

23. Lynch BM (2010) Sedentary behavior and cancer: a systematic review of the literature and proposed biological mechanisms. Cancer Epidemiol Biomarkers Prev 19, 2691-2709.

24. Cong YJ, Gan Y, Sun HL, et al. (2014) Association of sedentary behaviour with colon and rectal cancer: a meta-analysis of observational studies. Br J Cancer 110, 817-826.

25. Howard RA, Freedman DM, Park Y, et al. (2008) Physical activity, sedentary behavior, and the risk of colon and rectal cancer in the NIH-AARP Diet and Health Study. Cancer Causes Control 19, 939-953

26. Romaguera D, Vergnaud AC, Peeters PH, et al. (2012) Is concordance with World Cancer Research Fund/American
Institute for Cancer Research guidelines for cancer prevention related to subsequent risk of cancer? Results from the EPIC study. Am J Clin Nutr 96, 150-163.

27. van Lee L, Geelen A, van Huysduynen EJ, et al. (2012) The Dutch Healthy Diet index (DHD-index): an instrument to measure adherence to the Dutch Guidelines for a Healthy Diet. Nutr J 11, 49.

28. Aaronson NK, Ahmedzai S, Bergman B, et al. (1993) The European Organization for Research and Treatment of Cancer QLQ-C30: a quality-of-life instrument for use in international clinical trials in oncology. J Natl Cancer Inst 85, 365-376.

29. Vercoulen JH, Swanink CM, Fennis JF, et al. (1994) Dimensional assessment of chronic fatigue syndrome. J Psychosom Res 38, 383-392.

30. Vercoulen JH, Hommes OR, Swanink CM, et al. (1996) The measurement of fatigue in patients with multiple sclerosis. A multidimensional comparison with patients with chronic fatigue syndrome and healthy subjects. Arch Neurol 53, 642-649.

31. Zigmond AS \& Snaith R (1983) The hospital anxiety and depression scale. Acta Psychiatr Scanc 6, 10.

32. Sangha O, Stucki G, Liang MH, et al. (2003) The SelfAdministered Comorbidity Questionnaire: a new method to assess comorbidity for clinical and health services research. Arthritis Rheum 49, 156-163.

33. Van Roekel EH, Bours MJ, Breedveld-Peters JJ, et al. (2015) Light physical activity is associated with quality of life after colorectal cancer. Med Sci Sports Exerc 47, 2493-2503.

34. Wilson Van Voorhis CR \& Morgan BL (2007) Understanding power and rules of thumb for determining sample size. Tutor Quant Methods Psychol 3, 8.

35. van Roekel EH, Bours MJ, Breedveld-Peters JJ, et al. (2016) Modeling how substitution of sedentary behavior with standing or physical activity is associated with health-related quality of life in colorectal cancer survivors. Cancer Causes Control 27, 513-525.

36. Jansen M (2015) Op zoek naar de Limburg-factor. De gezondheidssituatie in Limburg onderzocht en verklaard. (Looking for the Limburg-factor. Investigation and explanation of the state of health in the province of Limburg). GGD Zuid Limburg, 2015. https://www.ggdghorkennisnet.nl/nieuws/4848-waarom-zijnlimburgers-ongezonder. (accessed May 2017).

37. Inoue-Choi $\mathrm{M}$, Lazovich $\mathrm{D}$, Prizment $\mathrm{AE}$, et al. (2013) Adherence to the World Cancer Research Fund/American Institute for Cancer Research recommendations for cancer prevention is associated with better health-related quality of life among elderly female cancer survivors. J Clin Oncol 31, $1758-1766$. 